\title{
Conservación, desarrollo y descentralización: lecciones de un modelo de gestión territorial público-privado en el sur de Chile
}

\section{Conservation, development and decentralization: Lessons from a public-private territorial management model in Southern Chile}

Francisca Vergara-Pinto ${ }^{a}$, Natalia Campos Albornoz ${ }^{b}$

a Universidad de Los Lagos, Chile.franverggara@gmail.com

b Universidad San Sebastián, Chile.nati.camposalbornoz@gmail.com

\section{RESUMEN}

Este estudio aborda la implementación del Sistema Regional de Áreas Protegidas (SIRAP), estrategia concebida con el objetivo de mejorar la representatividad de ecosistemas vulnerables en paisajes rurales y descentralizar la gestión de las áreas protegidas en Chile, esta vez considerando aquellos ecosistemas situados en predios privados. El objetivo consistió en conocer cuáles son los desafíos que enfrentan los territorios locales para implementar este tipo de categoría y qué gobernanzas son las más adecuadas para una gestión eficiente de estas áreas. A través de una revisión bibliográfica y análisis documental, se analizaron los resultados del proceso de descentralización llevado a cabo por el SIRAP en dos comunas de la región de Los Ríos (Chile), en donde esta estrategia se materializó y se tradujo en la creación de una nueva categoría de área protegida denominada Paisaje de Conservación Valle Río San Pedro, que incluye la creación de un "Consejo de Desarrollo" y una "Asociación de Municipalidades Paisajes de Conservación para la Biodiversidad de la región de Los Ríos”.

Palabras clave: Descentralización; conservación; desarrolloterritorial; gobiernos locales; participación

\section{ABSTRACT}

This study addresses the implementation of the Regional System of Protected Areas (SIRAP), a strategy designed with the aim of improving the representativeness of vulnerable ecosystems in rural landscapes and decentralizing the management of protected areas in Chile, this time, considering those ecosystems located on private lands. The objective was to know what were the challenges faced by local territories to implement this type of category, and which governances are best suited for efficient management of these areas. Through a bibliographic review and documentary analysis, the results of the decentralization process carried out by SIRAP were analyzed in two communes in the Los Ríos region (Chile), where this strategy materialized and resulted in the creation of a new category of protected area called Landscape of Conservation Valle Río San Pedro, which includes the creation of a "Development Council" and an "Association of Municipalities Conservation Landscapes for biodiversity of the region of Los Ríos".

Keywords: Decentralization; conservation; territorial development; local governments; participation 
Vergara \& Campos | Conservación, desarrollo y descentralización

\section{INTRODUCCIÓN}

La fragmentación de los ecosistemas de bosque nativo en el sur de Chile, ocasionada en gran medida durante el siglo XIX y XX por la ampliación de la frontera agrícola, la industria maderera y la sustitución por monocultivo forestal (Otero, 2006; Frank y Finckh, 1997), se ha convertido en objeto de interés de diversos actores sociales durante las últimas dos décadas. Ello se debe principalmente a la profundización de dos tensiones que enfrenta actualmente el bosque nativo: la preocupación por la disminución de biodiversidad ocasionada por la fragmentación de diversos hábitats (Lara et al., 2012; AIFBN, 2010; Manzur, 2005); y el interés puesto en los bosques como productores de beneficios para la sociedad, y por los valores que algunos grupos sociales le atribuyen a nivel identitario y territorial (Tecklin y Catalán, 2005; Oyarzún, Nahuelhual y Núñez, 2005). A lo anterior se suma, como una preocupación de alcance global, el hecho de que tales ecosistemas son representativos a nivel mundial, estando situados dentro de la Ecorregión del Bosque Templado Valdiviano (Dinerstein et al., 1995) y el Hotspot "Chilean Winter Rainfall - Valdivians Forests” (Arroyo et al., 2008). En respuesta, la conservación de ecosistemas de bosque nativo fragmentados por la intervención antrópica, como objeto de interés ha sido incorporado a la agenda de la comunidad científica, movimientos ciudadanos, instituciones no gubernamentales internacionales, y del Estado.

En este escenario, el Estado en sus distintos niveles de gobierno ha debido asumir un rol clave en la conservación, por ejemplo, mediante la promulgación de leyes y la creación de instituciones que gestionan el acceso a áreas protegidas, a través de un Sistema Nacional de Áreas Protegidas del Estado (SNASPE, creado mediante la Ley $\mathrm{N}^{\circ} 18.362$ de 1984). No obstante, ha debido transitar también hacia la promoción de instancias nuevas de interacción público-privada que posibiliten la conservación en áreas biodiversas simultáneamente habitadas por población humana, o por propietarios privados, debiendo prestar atención entonces a la forma de incorporar la conservación en los planes de desarrollo territorial. Una de las estrategias más recientes que busca responder a este desafío consiste en la creación de un Sistema Regional de Áreas Protegidas (SIRAP) enfocado en espacios biodiversos con singularidades territoriales, al estar localizados en predios privados (PNUD GEF SIRAP, 2013a; CONAMA GEF SIRAP, 2010), con el propósito de "implementar en dicho espacio, un modelo de gestión territorial compartido, basado en las necesidades e intereses del conjunto de actores que habita el territorio y en la protección de la biodiversidad... con el objetivo de mejorar la representatividad y descentralizar la gestión de las áreas protegidas" (PNUD GEF SIRAP, 2013b, p.1).

Al declararse como un proyecto descentralizador, el SIRAP se propone reformular la tradicional manera de gestionar los bienes de uso común, buscando incorporar aquellos bienes (ecosistemas) que escapan del dominio fiscal, mediante un diseño de gestión territorial compartido. De aquí se pueden desprender múltiples preguntas en torno a los procesos sobrellevados por el SIRAP, no obstante, este artículo se concentrará en atender una pregunta relacionada con la viabilidad de este tipo de estrategias en materia de conservación articulada al desarrollo territorial. Es decir, en la práctica ¿̇cómo se manifiesta a niveles subnacionales el desempeño de la descentralización en la gestión de la conservación y desarrollo territorial local? Para 
abordar lo anterior se propone como objetivo conocer cuáles son los desafíos que enfrentan los territorios locales para implementar este tipo de categoría, y qué gobernanzas son las más adecuadas para una gestión eficiente de estas áreas.

$\mathrm{Al}$ respecto, como hipótesis se sostiene que la descentralización de la gestión de la conservación articulada al desarrollo territorial local demanda una consideración fundamental a niveles subnacionales, especialmente a nivel de gobierno local: la transferencia de competencias en materia de áreas protegidas. Estas competencias dependerán de la interrelación de dos principales factores, a saber, de a) las capacidades institucionales y presupuestarias y b) los recursos humanos de las municipalidades. No obstante, tal consideración debe hallar eco en los territorios mediante la activación de conocimientos y valores locales para una apropiación de la estrategia a largo plazo, lo cual hace necesario considerar otros factores como c) los contextos socio-territoriales de las comunas (en donde se sitúan los ecosistemas no representados en unidades de conservación nacionales) y d) una participación activa de las poblaciones locales y propietarios privados. Según el grado de interrelación de estos cuatro factores, se manifestará la efectividad del SIRAP como modelo descentralizado en el ámbito.

Figura 1. Mapa representativo de los sitios prioritarios para la conservación de la biodiversidad de la región de Los Ríos, al año 2010. En el centro, el área-objetivo del SIRAP.

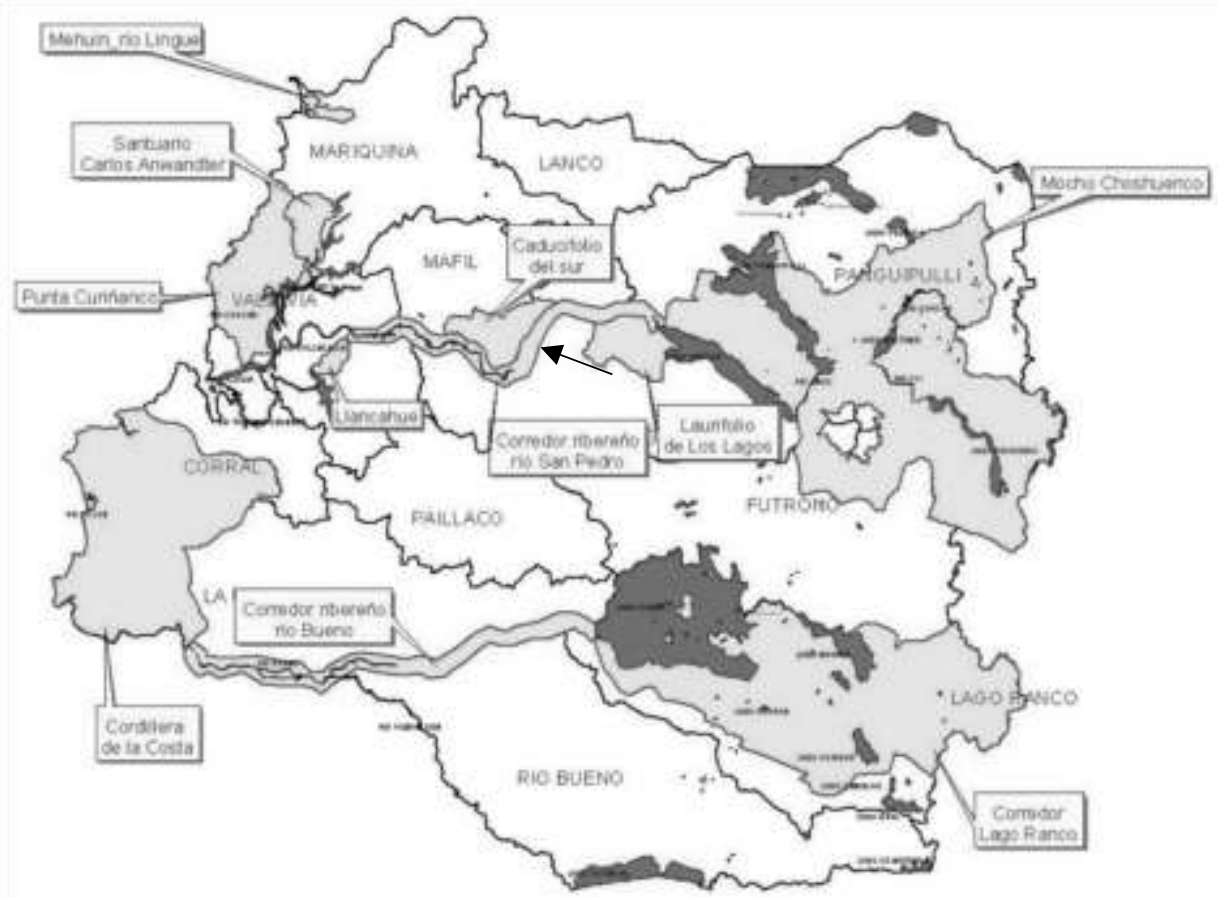

Fuente: Paisaje de Conservación Valle Río San Pedro.

Para abordar esta temática, el artículo comienza presentando una definición de los conceptos de desarrollo territorial local y descentralización, indicando a su vez la forma en que la conservación se articula a ellos. Luego, se presentan antecedentes recopilados desde fuentes secundarias a través de análisis documental (Sandoval, 2002), considerando una revisión bibliográfica para documentar aspectos generales 
en torno al proceso de implementación del SIRAP, enfocando la lente en los mecanismos desarrollados y sus resultados a nivel empírico. Lo anterior, por un lado, a modo de comprender las diferencias entre el modelo tradicional de conservación y la apuesta del SIRAP como un modelo de gestión territorial compartido, resultado de un proceso de descentralización de la gestión de las áreas protegidas que, por su naturaleza (conservar ecosistemas contenidos en predios privados), debe articularse al proceso de desarrollo territorial local. Asimismo se consideran los desafíos que enfrentan los territorios locales para implementar estas nuevas categorías de conservación y las gobernanzas más adecuadas para una gestión eficiente de estas áreas. Se finaliza con las principales conclusiones.

\section{DESARROLLO TERRITORIAL, CONSERVACIÓN Y DESCENTRALIZACIÓN: APROXIMACIÓN CONCEPTUAL}

El desarrollo territorial es un concepto que posee múltiples definiciones según las perspectivas teóricas desde donde se aborda. Por ejemplo, la definición de la Comisión Económica para América Latina y el Caribe (CEPAL) señala que este es "un proceso de construcción social del entorno, impulsado por la interacción entre las características geofísicas, las iniciativas individuales y colectivas de distintos actores y la operación de las fuerzas económicas, tecnológicas, sociopolíticas, culturales y ambientales en el territorio" (fuente: www.cepal.org). En cuanto a su dimensión económica, Serrano (2010) indica que "el potencial del desarrollo territorial se asocia a la idea de encadenamientos productivos y al concepto de una 'nueva política industrial' capaz de generar externalidades positivas en el entorno, asociadas a las circunstancias de proximidad. A la vez, la perspectiva territorial moviliza los activos presentes en el territorio agregando valor al conjunto de los procesos" (Serrano, 2010, p. 1). Tomando ambas definiciones en consideración, cabe añadir una tercera desarrollada por Vásquez-Barquero (2009), quien enfatiza que "el desarrollo de los territorios (...) depende por una parte de la capacidad que tengan los actores locales de identificar las condiciones humanas, sociales y productivas instaladas, y por otra de que existan las condiciones para poner en marcha una estrategia de largo plazo orientada a mejorar el bienestar general de las personas que habitan en ese territorio" (Vásquez-Barquero, 2009, citado en Thayer, 2011, p. 267).

El desarrollo en territorios que son biodiversos, además de habitados y productivos, se encuentra con una dimensión del medio biofísico a considerar, ya no solo en términos de recurso natural o actividad productiva, sino de relaciones ecosistémicas y relaciones socio-ecológicas. Reformulando la idea planteada en la cita anterior, se puede decir, entonces, que el desarrollo de los territorios biodiversos va a depender de la capacidad que tengan los actores locales de identificar las condiciones humanas, sociales, productivas y ecosistémicas del lugar que habitan, y por otra de que existan las condiciones para poner en marcha una estrategia de largo plazo orientada tanto a mejorar el bienestar de las personas que habitan en ese territorio, como la protección de los ecosistemas presentes en él. En cuanto al concepto de conservación de la biodiversidad, en este artículo se entiende según lo planteado por Tacón (2004), en tanto proceso que releva la importancia de la biodiversidad para las sociedades ya sea desde el punto de vista económico, cultural, ecológico o genético; así como el impacto de las sociedades en la biodiversidad. Por 
ejemplo, su pérdida puede limitar la disponibilidad de especies de importancia alimenticia o productiva, o afectar procesos de mayor escala -tanto espacial como temporal-intergeneracional- como la calidad del agua o estabilidad climática. El autor indica que los objetivos de la conservación se explican mediante tres principios fundamentales: 1) la mantención del cambio evolutivo y ambiental, 2) la mantención de la dinámica ecológica y 3) la consideración de la presencia humana. En torno a este último, señala que "la sociedad humana siempre ha tenido impactos sobre las comunidades ecológicas. [Por tanto] La conservación debe incorporarnos [como sociedad] para comprender los procesos naturales y diseñar e implementar soluciones" (Tacón, 2004, p. 18).

De ello se desprende la necesaria articulación que debe construirse entre estrategias de conservación y la planificación de un desarrollo territorial que atiende a los requerimientos tanto de la sociedad como de los ecosistemas que, de una u otra forma, la sociedad necesita y que también impacta. Frente a este panorama, no puede elevarse solo un discurso conservacionista ya que, en palabras de Campos (2010), este "ha sido criticado en múltiples ocasiones por la falta de propuestas para lograr el desarrollo del territorio, en el que existen prácticas de producción tradicionales y en lugares en los que, principalmente, [se] desarrollan actividades extractivas" (Campos, 2010, p. 58). Surgen alternativas al respecto, como aquella planteada por el Fondo Mundial para el Medio Ambiente (Global Environment Facility o GEF por su sigla en inglés) en conjunto con el Ministerio del Medio Ambiente (MMA): el SIRAP, estrategia cuyo objetivo es descentralizar la gestión de las áreas protegidas en Chile, en relación con el SNASPE. Pero ¿qqué significa descentralizar tal gestión? Para comprender ello, es necesario definir qué es la descentralización y cómo puede relacionarse con la conservación.

En términos conceptuales, se entiende la descentralización como "un proceso de políticas públicas que en su conjunto traspasan responsabilidades, recursos o autoridad desde el gobierno central a los gobiernos subnacionales, y donde estos últimos están dotados de autonomía" (Falleti, 2005; Rodden, 2004, citado en Vial y Hernández, 2017, p. 7). En el marco de un proyecto nacional descentralizador, según Boisier (2004), su implementación a escala territorial viene mediada por la confluencia de dos procesos. A saber, el cambio en las funciones reguladoras del gobierno nacional a través de la entrega de funciones relativas al desarrollo a los entes territoriales, y paralelo a ello el cambio en las percepciones de los propios territorios "de ser ellos los responsables de activar los varios mecanismos que operan actualmente como factores causales (...) de su desarrollo, y de su posicionamiento en la globalización" (Boisier, 2004, p. 33). Esto último, no obstante, puede verse obstaculizado por la ausencia de una 'acumulación de prácticas históricas' a causa de la rapidez del cambio social. Por otro lado, el proceso de descentralización y el traspaso de competencias desde el nivel nacional a los subnacionales, puede desplegarse de manera efectiva en contextos en los cuales este se articula con "la construcción de capacidades en el nivel territorial y la coordinación de acciones entre los distintos niveles de gobierno" (Vial y Hernández, 2017, p. 10).

En el contexto nacional, señala Delamaza (2013), la creciente demanda por descentralización y autonomía regional está siendo reconocida en la sociedad. Siendo indispensable el proceso de reforma institucional del Estado y las transferencias de competencias a niveles subnacionales, ello necesariamente "debe 
acompañarse de la modificación de las interrelaciones entre los actores sociales, la institucionalidad y los agentes políticos que pueden conducir las transformaciones desde los territorios" (Delamaza, 2013, p. 2). En materia de conservación, si bien las áreas protegidas del SNASPE son administradas descentralizadamente por la Corporación Nacional Forestal (CONAF), su declaración como tal es centralizada restándole así atribuciones a las regiones (Maturana et al., 2017). A esta situación se suma una serie de problemas que enfrentan distintas unidades o categorías de conservación del SNASPE, en cuanto a limitaciones de gestión y a la falta de actualización de los planes de manejo (Ibid). Además, los beneficios económicos obtenidos en las áreas protegidas - las cuales se sitúan principalmente en las áreas rurales del país -, ya sea por la recaudación de entradas, las actividades turísticas o las concesiones al interior de estos espacios, "están totalmente centralizadas, tanto en la dirección regional de CONAF como en su nivel nacional" (Maturana et al., 2017, p. 191). Por último, cabe destacar que este proceso de descentralización en Chile no constituye un caso aislado. Es sabido que en América Latina, desde hace un par de décadas, la problemática de la disminución de biodiversidad a causa del avance del extractivismo y del crecimiento urbano ha provocado debates en la sociedad, y a nivel de gobiernos centrales ha incidido en la implementación de normativas y leyes y en la adhesión a marcos normativos de carácter global. No obstante, el debate no ha producido los mismos resultados en todos los países, hallándose casos (e.g. en Perú o Colombia) que ilustran el uso mínimo o nulo de categorías de manejo creadas para el nivel regional (Castaño, 2008).

\section{EL SIRAP EN LA PRÁCTICA: DECISIONES, VOLUNTADES Y PROYECCIONES}

\subsection{Decisiones: delimitación y creación de una nueva área protegida}

Como se señaló anteriormente, varios de los ecosistemas terrestres protegidos en Chile a través del SNASPE, corresponden a ecosistemas que conforman la ecorregión de bosque templado valdiviano. No obstante, el sistema posee importantes vacíos en cuanto a su representación de la biodiversidad excluyendo algunos ecosistemas y, por tanto, dejando hábitats sin protección por parte del Estado (Tecklin y Catalán, 2005). Dos de aquellos corresponden a los ecosistemas de bosque Caducifolio del Sur y Laurifolio de Los Lagos, bosques que se sitúan en la depresión intermedia de la región de Los Ríos, en la subcuenca del río San Pedro (comunas de Los Lagos y Máfil), en territorios rurales habitados por poblaciones que desarrollan distintas actividades productivas, principalmente relacionadas con el ámbito agrícola, ganadero, forestal y turístico (Municipalidad de Los Lagos, 2013).

Como respuesta a esta situación, y dado que la presencia de ambos ecosistemas posicionó este territorio como uno de los 10 sitios de alto valor de biodiversidad de la región, entre los años 2008 y 2013 se implementa el proyecto GEF-SIRAP. Este proyecto ha focalizado sus esfuerzos de intervención principalmente en la Cordillera de la Costa y el valle central de la región de los Ríos, y en la Cordillera de la costa y la precordillera andina de la región de Los Lagos (GEF SIRAP, 2013a). Como estrategia, comienza siendo desarrollado por la Comisión Nacional del Medio Ambiente (CONAMA) y luego por el Ministerio del Medio 
Ambiente (MMA), con apoyo internacional del Fondo Mundial para el Medio Ambiente (GEF) y del Programa de las Naciones Unidas para el Desarrollo (PNUD). Luego queda instalado en la gestión municipal de dos comunas, Los Lagos y Máfil, traduciéndose en una nueva categoría de conservación denominada "Paisaje de Conservación Valle Río San Pedro" (PCVRSP). La creación de esta categoría se traduce en el principal resultado del proyecto SIRAP que sostiene la creación de posteriores procesos de asociatividad y reformulación de los planes de desarrollo comunal. La categoría Paisaje de conservación se refiere a "un territorio habitado que posee un patrimonio natural y cultural de interés regional y/o nacional, delimitado y gestionado con el propósito de implementar una estrategia consensuada y efectiva de conservación y desarrollo, que permita mantener y/o mejorar los valores de interés del territorio y contribuir al mejoramiento de la calidad de vida de la población" (PNUD GEF SIRAP, 2013a, p. 7). Esta nueva categoría de área protegida utiliza como referencia la categoría V "Paisaje terrestre/marino protegido" propuesta por la Unión Internacional para la Conservación de la Naturaleza (UICN), diferenciándose de otras áreas protegidas (e.g. reserva o parque nacional) por considerar la protección de ecosistemas inmersos en matrices culturales como también productivas, que además proveen de servicios ecosistémicos a la población, dando respuesta así a la necesidad por parte de la sociedad local de comprender los procesos naturales de su entorno e implementar soluciones ante la fragilidad a la que están expuestos los ecosistemas (Tacón, 2004).

Quienes participan en este proyecto corresponden a propietarios de predios privados, organizaciones territoriales, además de servicios públicos y empresas privadas que contienen patrimonio de bosque nativo dentro de sus propiedades (Municipalidad de Los Lagos, 2013). En la creación efectiva de una nueva categoría de área protegida, independiente del SNASPE, se puede observar una primera manifestación del proceso de descentralización impulsado por el SIRAP en tanto la gestión del área protegida se desprende del sistema nacional, instalándose a nivel municipal de manera autónoma.

Figura 2. Mapa territorio Paisaje de Conservación Valle Río San Pedro, situado entre las comunas de Los Lagos y Máfil, Provincia de Valdivia.

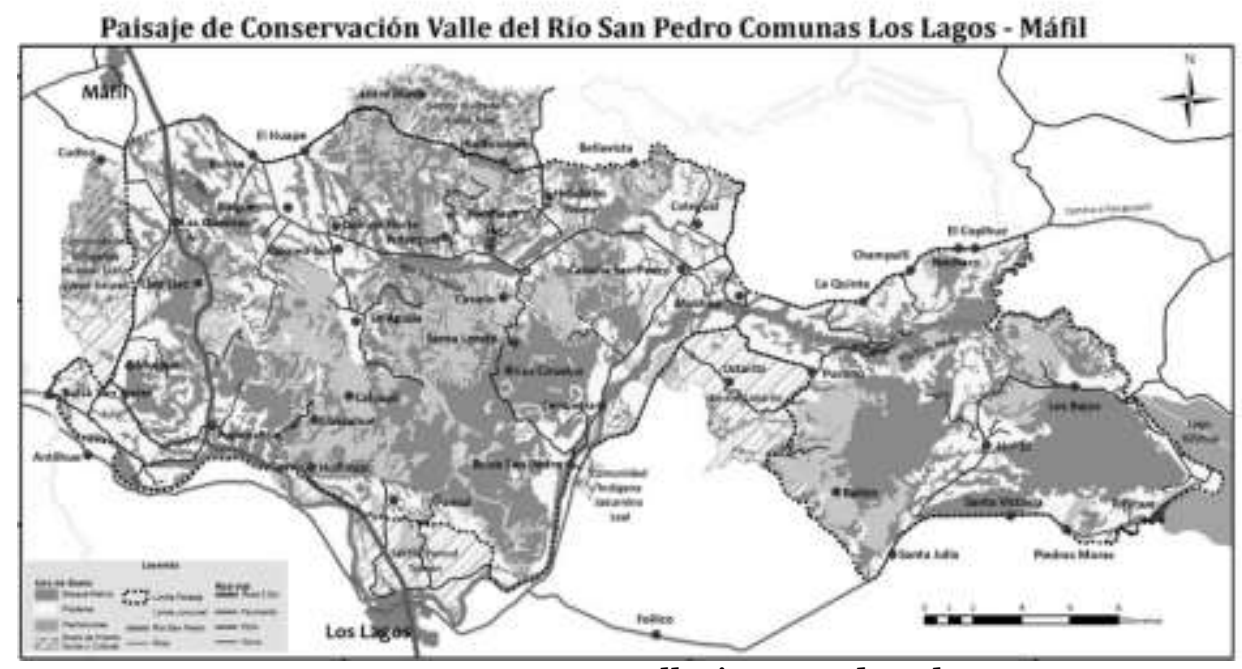

Fuente: www.valleriosanpedro.cl. 


\subsection{Voluntades: Sociedad y gobierno local articulados}

A partir del PCVRSP son creadas otras dos figuras que se institucionalizan a nivel local, pero que nacen de la articulación público-privada y se sostienen en la participación activa de los actores locales: el Consejo de Desarrollo y la Asociación de Municipalidades. Una tercera figura tiene relación con la reformulación del Plan de Desarrollo Local (PLADECO) de ambas comunas, a propósito de la incorporación del enfoque de conservación y la cogestión de la nueva área protegida a escala bi-comunal.

El Consejo de Desarrollo y la Asociación de Municipalidades están estrechamente vinculados, aunque mantienen funciones diferentes, constituyéndose en espacios para la toma de decisiones consensuadas entre todos los actores públicoprivados. Por su parte, la conformación del Consejo de Desarrollo "permitió fortalecer la base de institucionalidad pública del modelo de gestión territorial, lo que se tradujo en la formalización de la Asociación de Municipios de Los Lagos y Máfil” (PNUD GEF SIRAP, 2013a, p. 17), a partirdeuna articulación público-privada.

El Consejo de Desarrollo, en su proceso de formación, fue constituido por una diversidad de actores (Cuadro 1) con presencia en el territorio, reunidos en torno a tres objetivos clave: lograr que personas y organizaciones se conozcan y acuerden trabajar juntos; elaborar estatutos de funcionamiento que incluyan una visión y misión común para el territorio; crear y acordar un Plan de Trabajo enfocado en establecer y gestionar el territorio del PCVRSP, de forma sostenible y consensuada (ibid. 2013, p. 9). En esta articulación público-privada y asociativa que el PCVRSP buscó y logró establecer, se puede observar una segunda manifestación del proceso de descentralización impulsado por el SIRAP.

La creación de ambos espacios evidencia un proceso de creatividad organizacional, que resulta del esfuerzo por incorporar la lógica de la conservación dentro de las dinámicas económico-productivas de los actores locales involucrados, quienes "aprendieron a mirar y ver más allá de su predio: ver el territorio como un ecosistema integrado, más allá de los límites del mapa, y buscar los beneficios para el territorio en su conjunto" (Ibid, p. 19). Con ello se consolida la articulación de dos municipalidades, Los Lagos y Máfil, cuyos alcaldes "firmaron la creación de la 'Asociación de Municipios Paisajes de Conservación para la biodiversidad de la Región de Los Ríos', que les permitirá trabajar en conjunto diversas temáticas con el fin de proteger y fortalecer su patrimonio natural" (Ibid, p. 21).

Junto a ambos espacios, se logró la incorporación de las temáticas de biodiversidad (y con ello el enfoque de conservación) en los Planes de Desarrollo Comunal (PLADECO) y la creación de oficinas técnicas en ambos municipios, siendo los equipos municipales capacitados mediante el SIRAP, constituyendo aquello una tercera manifestación del proceso de descentralización del SIRAP a nivel de gobierno local. El PLADECO, según la Ley $\mathrm{N}^{\circ} 18.695$ Orgánica Constitucional de Municipalidades, es un instrumento de planificación de carácter indicativo, "rector del desarrollo en la comuna, [que] contemplará las acciones orientadas a satisfacer las necesidades de la comunidad local y promover su avance social, económico y cultural. Su vigencia mínima será de cuatro años, sin que necesariamente deba coincidir con el periodo de desempeño de las autoridades municipales electas por la ciudadanía”. 
Cuadro 1: Actores que conforman el Consejo de Desarrollo del PCVRSP, año 2018.

\begin{tabular}{|l|l|l|}
\hline Tipo de actor & $\begin{array}{l}\text { Representantes en el Consejo } \\
\text { de Desarrollo }\end{array}$ & Actores \\
\hline $\begin{array}{l}\text { Organización } \\
\text { social }\end{array}$ & $\begin{array}{l}\text { Presidentes de las } \\
\text { organizaciones }\end{array}$ & $\begin{array}{l}\text { 9 organizaciones } \\
\text { juntas de vecinos } \\
\text { comités de agua potable rural } \\
\text { comité de salud } \\
\text { 1 agrupación de mujeres }\end{array}$ \\
\hline $\begin{array}{l}\text { Comunidad } \\
\text { indígena }\end{array}$ & Presidente de la comunidad & comunidad indígena \\
\hline $\begin{array}{l}\text { Empresa } \\
\text { privada }\end{array}$ & $\begin{array}{l}\text { Encargado de área ambiental } \\
\text { y/o social }\end{array}$ & $\begin{array}{l}\text { 2 empresas forestales (Forestal } \\
\text { Valdivia, Hancock) }\end{array}$ \\
\hline $\begin{array}{l}\text { Ejército de } \\
\text { Chile }\end{array}$ & $\begin{array}{l}\text { Designado en representación } \\
\text { de la institución }\end{array}$ & $\begin{array}{l}\text { 2 predios haras, dedicados a la } \\
\text { crianza de animales }\end{array}$ \\
\hline $\begin{array}{l}\text { Medio } \\
\text { Ambiente }\end{array}$ & Seremi o un representante & Servicio público \\
\hline Energía & Seremi o un representante & Servicio público \\
\hline MINAGRI & Seremi o un representante & Servicio público \\
\hline SAG & Director o un representante & Servicio público \\
\hline INDAP & Director o un representante & Servicio público \\
\hline CONAF & Director o un representante & Servicio público \\
\hline Salud & Director o un representante & Servicio público \\
\hline Subdere & Jefe regional & Servicio público \\
\hline GORE & $\begin{array}{l}\text { Jefe de división territorial o } \\
\text { un representante }\end{array}$ & Servicio público \\
\hline $\begin{array}{l}\text { Corporación } \\
\text { de Fomento } \\
\text { Productivo }\end{array}$ & Gerente & Servicio público \\
\hline
\end{tabular}

Fuente: Consejo de Desarrollo del PCVRSP.

La lógica que integra la conservación de la biodiversidad con el desarrollo territorial pasa a ser apropiada por el gobierno local y pasa a formar parte de la hoja de ruta no solo de una sino dos municipalidades en la región de Los Ríos. En este proceso se puede identificar una transformación en la visión y definición del territorio, resignificando los ecosistemas como proveedores de bienestar incluso a nivel intergeneracional, lo que se traduce en un cambio a nivel económicoproductivo que no necesariamente prohíbe sino que abre otras oportunidades económicas y potencia las interrelaciones entre la sociedad local e instituciones. De esta forma, es posible reconocer en la gestión descentralizada del SIRAP, a través de las figuras creadas en su implementación, una noción acerca de que "en el territorio se sintetizan, transforman y reinterpretan las trayectorias de las personas y de su entorno, las dinámicas de mercado y la presencia institucional" (Serrano, 2010). En este sentido, las tres figuras creadas en el marco del SIRAP para gestionar el Paisaje de Conservación, han sido articuladas para operar a nivel de la planificación bicomunal, potenciando entre la sociedad local la valoración de los ecosistemas y entre los actores institucionales que deben tomar decisiones respecto al manejo presente y futuro del territorio. 
Pese al éxito de la experiencia, la implementación de esta nueva figura de conservación no ha estado exenta de tensiones. Algunas de ellas se relacionan con: la focalización territorial en función de áreas de alto valor de conservación, considerando que discrepa de la forma en que trabajan las municipalidades, a través de asistencias técnicas menos personalizadas y atendiendo a toda la comuna; incorporan en el quehacer cotidiano $\mathrm{y}$ en la planificación comunal una discriminación positiva hacia el territorio, en función de sus atributos de biodiversidad y el modelo de gobernanza operante; una gran rotación de personal que fragiliza las confianzas, dificultando la continuidad de los procesos; y finalmente; el funcionamiento de diversas figuras operacionales conectadas, pero con algún grado de autonomía como son: la Asociación de Municipalidades, las Municipalidades de Los Lagos y Máfil, el Consejo de Desarrollo y la Asociación de Productores certificados del Paisaje de Conservación.

\subsection{Proyecciones: EI SIRAP como modelo descentralizado de gestión de la conservación y el PCVRSP como modelo de gestión territorial compartida}

La gestión llevada a cabo por el PCVRSP ha tenido resultados que han sido observados por actores de otras regiones del país, posicionándose como modelo a seguir por organizaciones e instituciones interesadas en integrar la conservación y el desarrollo territorial dentro de sus comunas. Ejemplo de ello son los 5 nuevos Paisajes de Conservación creados en las regiones de Los Lagos y Metropolitana, donde se han replicado desde el año 2013 a la fecha el modelo de gobernanza y las unidades demostrativas del PCVRSP.

El año 2013, en el marco de la ejecución del Proyecto GEF Sistema Nacional de Áreas Protegidas (SNAP), el Ministerio del Medio Ambiente en alianza con la Municipalidad de Alhué diseñaron e implementaron el Paisaje de Conservación de Alhué y lo definieron como "una interacción entre elementos naturales (árboles nativos, arbustos, ríos, bosques, agua, rocas y piedras) y elementos humanos (construcciones típicas, campos agrícolas, vegetación introducida, caminos, iconos simbólicos, mitos, leyendas y costumbres)" (Centro de Estudios del Desarrollo, 2014: 4). Agregaban que, "este es un territorio con un patrimonio natural y cultural altamente valorado por los habitantes de la comuna y de gran importancia para la Región Metropolitana de Santiago y para el país. Su identidad y atributos representan oportunidades de bienestar humano y de desarrollo sustentable, que requieren ser gestionados de manera integral y participativa para disminuir su vulnerabilidad ante la presión humana" (ibídem, 2014, p. 18).

En la Región de Los Lagos, el año 2013 se inicia la implementación del Paisaje de Conservación Chiloé, con apoyo del Ministerio del Medio Ambiente y financiamiento del Fondo Nacional de Desarrollo Regional (FNDR) de Los Lagos. Esta experiencia involucra las comunas de Ancud y Dalcahue, quienes lo definen como un "territorio/maritorio habitado, que posee valores naturales y culturales de interés regional y/o nacional, delimitado y gestionado con el propósito de implementar una estrategia consensuada y efectiva de conservación y desarrollo, que permita mantener y/o mejorar los valores antes señalados, contribuyendo de este modo a la calidad de vida de la población asegurando la base de recursos naturales y patrimoniales existentes" (Seremi deMedio Ambiente Región de Los Lagos, 2016, s/p). 
Por su parte, en el contexto de la ejecución del Proyecto GEF Corredores Biológicos de Montaña se crearon los Paisajes de Conservación: a) Islas y Cordones del Maipo; b) Cordilleras y c) Pucaras. Al revisar los diagnósticos territoriales elaborados el año 2014, se observa que las tres iniciativas son definidas como territorios que cuentan con una visión compartida, concertada y negociada con los diversos actores locales que participan del Consejo de Desarrollo, y que se expresa en un Acuerdo o Acta que constituye un compromiso para quienes lo firman y también para los servicios públicos que participan en él. El Acuerdo o Acta de constitución del Paisaje de Conservación debe contener el fin buscado y los compromisos de los firmantes, además de un Plan Estratégico que incluya una visión, misión, objetivos y metas a alcanzar en un mediano plazo.

En cada una de estas experiencias se promueve la participación de diversos actores del territorio para impulsar un objetivo planteado de forma consensuada. Estos actores locales, involucrados directamente en el territorio, le otorgan identidad al grupo a partir de sus diferencias socioculturales y socioeconómicas, entre otras, y son capaces de construir una visión en común con relación al uso y protección de los ecosistemas y al tipo de desarrollo que se proyecta para el territorio (PNUD GEF SIRAP, 2013a, p. 18).

En el interés puesto por actores de otros territorios, que observan en el SIRAP y el PCVRSP como modelos a seguir para replicar en los suyos, destaca un pilar fundamental sobre el que se sostienen estas iniciativas a nivel local: la participación. Si bien suele asociarse el concepto de participación al de participación ciudadana (que denota otro asunto distinto al tratado en este artículo), aquí se entiende como el substrato de la articulación público-privada construida en el territorio, siendo posible notar en ello lo que señala Delamaza (2017), en cuanto a que "los avances del proceso descentralizador pueden fortalecer la participación en la medida que, por una parte, acerquen las decisiones a los ciudadanos, que estos tengan oportunidades para tomar decisiones en los territorios y, por otra parte, que asuntos relevantes se decidan a nivel local o regional, es decir, que se pueda decidir sobre algo relevante" (Delamaza, 2017, p. 74).

Evidentemente, la efectividad de la implementación de un Sistema Regional de Áreas Protegidas, cuyo objeto (ecosistemas) está situado en manos de la gente (predios privados) y es transformado por diversos usos y valores (matrices culturales y productivas), se debe mucho al hecho de haber tomado en cuenta el contexto socioterritorial y la participación activa de la sociedad local, esta última potenciada por los espacios creados e institucionalizados por el gobierno local para dar soporte y sostenibilidad a la articulación entre actores que el SIRAP logró construir.

\section{CONSERVACIÓN Y DESARROLLO: HACIA UNA SÍNTESIS DE LA EXPERIENCIA DEL PCVRSP}

¿Cómo se manifiesta a niveles subnacionales el desempeño de la descentralización en la gestión de la conservación y desarrollo territorial? Con relación a la implementación de un Sistema Regional de Áreas Protegidas (SIRAP) en el sur de Chile, tal desempeño puede ser observado en las cuatro figuras que fueron descritas anteriormente, que influyeron en la transformación de la visión 
sobre los ecosistemas que están insertos en matrices culturales y productivas que parecerían incompatibles con la conservación:

- La creación de una nueva categoría de área protegida denominada Paisaje de Conservación, que no excluye las prácticas productivas de las personas que habitan el territorio, sino que intenta -y logra- incorporar un enfoque de conservación en aquellas prácticas en virtud de proteger los ecosistemas y asegurar los beneficios que estos brindan a la sociedad.

- La creación de un Consejo de Desarrollo y una Asociación de Municipalidades, como espacios institucionales para la toma de decisiones a escala local, pero a nivel bicomunal, considerando que las municipalidades y actores locales de dos comunas (Los Lagos y Máfil) decidieron reconocer que los ecosistemas presentes en sus territorios atraviesan los límites político-administrativos.

- La incorporación del enfoque de conservación en los Planes de Desarrollo Comunal (PLADECO) en ambas comunas, como se señala, por ejemplo, en el PLADECO de Los Lagos; "la temática medioambiental en la comuna está determinada por la constante preocupación desarrollada por dos estamentos que en su quehacer, han generado un posicionamiento transversal del ámbito, en los diferentes actores tanto públicos como privados insertos en la comunidad. De aquello, podemos señalar la eficiente labor del proyecto GEF SIRAP, los cuales han logrado desarrollar y posicionar el área de conservación como un nicho con múltiples potencialidades, integrando la protección del patrimonio natural al desarrollo sustentable" (Municipalidad de Los Lagos, 2013).

Estos constituyen los procesos más representativos del PCVRSP desde su fecha de conformación en 2013 a la actualidad. Su revisión da cuenta de la efectiva implementación de lo que Simonetti et al. (2002) denominan una cooperación público-privada orientada a la conservación de los ecosistemas de bosque nativo presentes en predios privados, cooperación materializada en las acciones de conservación llevadas a cabo por los actores locales dentro de sus actividades productivas. En este sentido, la integración de una lógica que potencia la conservación de la biodiversidad dentro de procesos de desarrollo territorial ha conducido a que se construyan relaciones sociales entre actores diversos -a nivel comunitario, interorganizacional, institucional, etc.- que antes de participar en el PCVRSP podían no tener vínculos a nivel territorial. Estos actores resignifican el territorio, aumentando con ello la riqueza del territorio (Juárez, 2013) al diversificar los significados asociados a la tierra, a los ecosistemas y a los bienes comunes, visualizando un beneficio no solo económico, sino ecológico, social y cultural, visible a través de las nuevas formas actitudinales, primero, y productivas, después, en torno al manejo de sus propios predios y de los ecosistemas contenidos dentro.

La tarea del SIRAP y del Paisaje de Conservación como materialización de la estrategia, implica una visión de los ecosistemas construida desde el conocimiento científico y los valores locales atribuidos; por un lado, transmitiendo hacia la sociedad local el rol socioecológico de la biodiversidad en sus dinámicas económicoproductivas, como la provisión de agua y productos forestales madereros y no madereros, entre otros; y por el otro, activando valores locales intangibles, por ejemplo, con sentido patrimonial (Vergara, 2017, Campos-Medina et al., 2018) o 
usos culturales tradicionales. Al mismo tiempo, el SIRAP ha debido reformular las clásicas relaciones verticales entre actores, en el sentido de que, esta vez, el accionar de las poblaciones locales, propietarios privados y gobiernos locales es clave y necesario en la conservación de la biodiversidad presente en áreas productivas.

Esta situación se suma a la condición actual de diversos ecosistemas que no están contenidos dentro de áreas protegidas por el SNASPE. Es en este contexto donde emerge y desde donde se puede comprender la implementación del SIRAP, pues la protección de los ecosistemas que quedan fuera del SNASPE, en primer lugar, hace necesario el diseño de una estrategia que permita la conservación in situ de ecosistemas presentes en paisajes rurales; en segundo lugar, requiere de la voluntad de quienes resultan ser los propietarios de los predios en donde estos se sitúan; y en tercer lugar, requiere de capacidades institucionales que sostengan en el mediano y largo plazo las acciones de conservación, articulándolas necesariamente a la planificación del desarrollo de los territorios.

\section{CONCLUSIONES}

$\mathrm{Al}$ respecto, el análisis de los resultados del SIRAP en la región de Los Ríos a partir de la experiencia del PCVRSP, permite concluir que, en sus distintos procesos y a través de la creación de sus distintas figuras, ha alcanzado su objetivo de descentralizar la gestión de las áreas protegidas hacia niveles subnacionales, sobre la base de fortalecer las capacidades institucionales y las competencias de los recursos humanos a nivel de gobierno local; y sobre la base de brindar un rol protagónico a los actores locales mediante la creación de espacios para la participación activa, para la toma de decisiones y la realización de acciones consensuadas en un territorio compartido. Se observan patrones cíclicos de actividad, con algunos espacios de intermitencia, propios de este tipo de iniciativas. Sin embargo, elementos como la diversidad de instituciones públicas y privadas que participan del modelo de gobernanza, sus participaciones constantes liderando acciones concretas a diferentes escalas y el apalancamiento de recursos públicos, que benefician principalmente a productores del territorio, generan presiones políticas, sociales y económicas que permiten continuar planificando acciones para armonizar la conservación con la producción.

Precisamente, lo que buscó construir y liderar el SIRAP ha sido un proceso de descentralización y reestructuración de la gestión de la conservación en territorios biodiversos y productivos, encontrándose ineludiblemente con la necesidad de participar en la planificación del desarrollo territorial en conjunto con los gobiernos locales y de construir capacidades entre todos los actores involucrados en el proceso. Frente al panorama que existe a nivel latinoamericano en materia de descentralización de gestión de la conservación, retomando a Castaño (2008), se puede decir que en Chile se ha creado una categoría de área protegida para el nivel regional, el paisaje de conservación, basada en un modelo de gestión territorial compartida, y su uso ha sido eficaz y vigente.

\section{BIBLIOGRAFÍA}

AIFBN. (2010), Informe nacional: Monitoreo forestal independiente en cuencas hidrográficas abastecedoras de agua en la XIV región de Los Ríos. Autor. 
Arroyo, M., Marquet, P., Marticorena, C. Simonetti, J. Cavieres, L. Squeo, F. Rozzi, R. y Massardo, F. (2008), "El hotspot chileno, prioridad mundial para la conservación". En: CONAMA, Biodiversidad de Chile, Patrimonio y Desafíos (pp.90-95). Chile: Ocho Libros Editores.

Boisier, S. (2004), "El desarrollo territorial y la descentralización en el lugar y en las manos de la gente". EURE, 30(90): 27-40.

Castaño, C. (2008), Manual de capacitación. Descentralización de la gestión de las áreas protegidas en América Latina. Programa FAO/OAPN, Red Latinoamericana de Cooperación técnica en Parques Nacionales, otras áreas protegidas, Flora y Fauna silvestre, España.

Centro de Estudios del Desarrollo. (2014). Guía técnica para la planificación participativa de paisajes de conservación y su integración en los planes de desarrollo comunal, Año 2014. Recuperado de http://bdrnap.mma.gob.cl/recursos/privados/Recursos/CNAP/GEFSNAP/CED_2014_b.pdf

Campos, N. (2010), Valoración socio-territorial de la biodiversidad. Un aporte para la incorporación al Plan de Desarrollo Comunal de Los Lagos. Región de Los Ríos. Tesis de grado, Universidad de Chile.

Campos-Medina, J., Vergara-Pinto, F., Echeverría, A., Contreras, P. y Abarzúa, A. (2018), "Resignificación del patrimonio paleontológico presente en el río San Pedro (Cuenca del río Valdivia, Chile)". PASOS Revista de Turismo y Patrimonio Cultural, 16(3): 655-670.

CONAMA, GEF SIRAP y PNUD. (2010), Estrategia de conservación de la biodiversidad de la región de Los Ríos, Ministerio del Medio Ambiente, Gobierno de Chile.

Delamaza, G (2013), Fortalecimiento de las Capacidades Regionales y Cohesión Territorial. Nueva Agenda Regional. Santiago: RIMISP.

(2017), “¿Participar para Descentralizar o Descentralizar para Participar?”. En: Vial, C. y Hernández, J (eds.), ¿Para qué Descentralizar? Centralismo y políticas públicas en Chile. Análisis y evaluación por sectores (pp. 73-96). Santiago de Chile: ICHEM.

Dinerstein, E. Olson, D. Graham, D. Webster, A. Primm, S. Bookbinder, M. y Ledec, G. (1995), Una evaluación del estado de conservación de las eco-regiones terrestres de América Latina y el Caribe, Estados Unidos: WWF y Banco Mundial.

Elbers, J. (Ed.) (2011), Las áreas protegidas de América Latina: situación actual y perspectivas para el futuro, Quito, Ecuador, UICN.

Frank, D. y Finckh, M. (1997), "Impactos de las plantaciones de pino oregón sobre la vegetación y el suelo en la zona centro-sur de Chile". Revista Chilena de Historia Natural, 70, 191-211.

Juárez, G. (2013), "Revisión del concepto de desarrollo local desde una perspectiva territorial". Revista Lider, 23: 9-28.

Lara, A. Solari, M. Prieto, M. y Peña, M. (2012), "Reconstrucción de la cobertura de la vegetación y uso del suelo hacia 1550 y sus cambios a 2007 en la ecorregión de los bosques valdivianos lluviosos de Chile $\left(35^{\circ}-43^{\circ} 30^{\prime} \mathrm{S}\right)$ ". Bosque 33(1): $13-23$. 
Manzur, M. (2005), Situación de la biodiversidad en Chile. Desafíos para la sustentabilidad. Programa Chile Sustentable. Chile: LOM.

Maturana, F., Fuenzalida, M., Arenas, F. y Henríquez, C (2017), "La planificación territorial en Chile y el proceso de descentralización”. En: Vial, C. y Hernández, J. (eds.), ¿Para qué Descentralizar? Centralismo y políticas públicas en Chile. Análisis y evaluación por sectores (pp. 181-208). Santiago: ICHEM.

Municipalidad de Los Lagos (2013), Plan de Desarrollo Comunal Los Lagos 20132017.

\section{(2013). Recuperado}

de http://www.muniloslagos.cl/transparencia/documentos/2014/6/33/Pladeco 2013_2017.pdf

Otero, L. (2006), La huella del fuego. Santiago de Chile: Pehuén Editores.

Oyarzún, C. Nahuelhual, L. y Núñez, D. (2005), "Los servicios ecosistémicos del bosque templado lluvioso: producción de agua y su valoración económica”. Ambiente y Desarrollo, 20(3) - 21(1), 88-95.

PNUD GEF SIRAP (2013a), Conformación de un Consejo de Desarrollo para la instalación de un modelo de gestión territorial compartida para la sostenibilidad. Recuperado de http://www.cl.undp.org/content/dam/chile/docs/medambiente/undp_cl_ medioambiente_co nformacion-de-un-consejo-de-desarrollo-para-lainstalacion-de-un-modelo.pdf?download

PNUD GEF SIRAP (2013b), Guía de procedimientos básicos para el diseño ecológico territorial de un paisaje de conservación. Un ejemplo aplicado a los bosques templados fragmentados del sur de Chile. Documento de trabajo Proyecto GEF SIRAP.

Sandoval, C. (2002). Investigación cualitativa. Bogotá: ARFO Editores.

Seremi de Medio Ambiente Región de Los Lagos. (2016). Programa Aplicación de un modelo de gestión para el manejo y uso sustentable del territorio noroeste de Chiloé: Un paisaje de conservación para la Región de Los Lagos. Documento de trabajo. Recuperado de https://www.muniancud.cl/inicio/wpcontent/uploads/2016/11/FNDR-PAISAJE-DE-CONSERVACI\%C $3 \% 93 \mathrm{~N}$ -

GOBERNACI\%C $3 \% 93 \mathrm{~N}-\mathrm{Y}-\mathrm{SSPP} . \mathrm{pdf}$

Serrano, C. (2010), Políticas de desarrollo territorial en Chile. RIMISP, Centro Latinoamericano de Desarrollo Rural.

Simonetti, J., Grez, A. y Bustamante, R. (2002), "El valor de la matriz en la conservación ambiental". Ambiente y Desarrollo, 18 (2, 3, 4): 116-118.

Tacón, A. (2004), Conceptos generales para la conservación de la biodiversidad. Programa de Fomento para la conservación de tierras privadas de la Décima región. Proyecto CIPMA-FMAM, Valdivia, Chile.

Tecklin, D. y Catalán, R. (2005), "La gestión comunitaria de los bosques nativos en el sur de Chile: situación actual y temas en discusión”. En: Catalán, R. et al, (eds.), Bosques y comunidades del sur de Chile (pp. 19-40). Santiago de Chile: Editorial Universitaria.

Thayer, L. (2011), "Descentralización y desarrollo regional en Chile. Una mirada desde la sociedad". Polis, 10(30): 267-287. 
Vergara \& Campos | Conservación, desarrollo y descentralización

Vergara, F. (2017), Bosques australes y sustentabilidad: una aproximación etnográfica a los significados del bosque nativo, en el Valle del Río San Pedro, Región de los Ríos. Tesis de grado, Universidad de Concepción.

Vial, C. y Hernández, J. (eds.) (2017), ¿Para qué descentralizar? Centralismo y políticas públicas en Chile. Análisis y evaluación. 